式中 $\left[\mathrm{Cu}^{2+}\right]_{\mathrm{T}}$ 表示透析前 $\mathrm{Cu}^{2+}$ 的总浓度, 由 EDTA 标定, $\left[\mathrm{Cu}^{2+}\right]_{0}$ 表示达到平衡后由 AAS 测 出的平衡浓度, $[\mathrm{P}]_{\mathrm{T}}$ 表示蛋白质的总浓度. 假定透析达到平衡后, 透析袋内外自由铜离子的 平衡浓度相等(实际上是化学势相等), $\bar{n}$ 可由透析袋内外溶液体积 (总体积) 和实验测得的 $\left[\mathrm{Cu}^{2+}\right]_{0}$ 计算得到的. 在 $\mathrm{Cu}$ (II )-HSA 中 $\bar{n}_{\text {max }}=2.19, \mathrm{Cu}\left(\right.$ II )-BSA 中 $\bar{n}_{\text {max }}=2.89$. 这表明两 体系中平均结合类型数分别为 2.19 和 2.89 .

从图 1 和图 2 可见, 4 个 HSA 浓度得到 4 条 $\bar{n}_{\mathrm{c}}$ 对 $\left[\mathrm{Cu}^{2+}\right]$ 的点绘曲线, 而对 BSA 体系则与 其浓度无关, 4 条曲线基本一致. 图 2 仅给出了 $2.0 \times 10^{-4} \mathrm{~mol} \cdot \mathrm{dm}^{-3}$ 浓度的曲线.

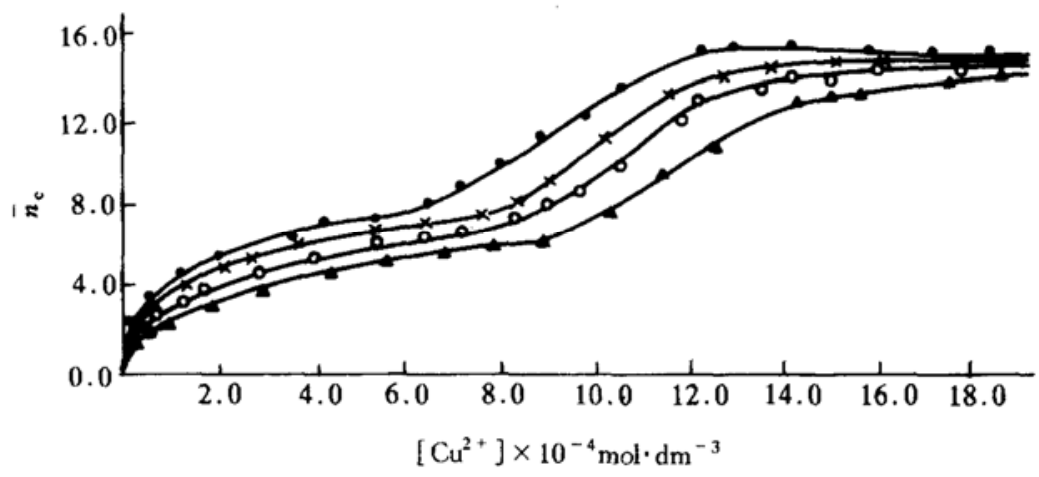

图 1 不同 $\mathrm{HSA}$ 浓度下 $\mathrm{Cu}\left(\right.$ II ) $-\mathrm{HSA}$ 的 $\vec{n}_{\mathrm{c}}$ 与 $\left[\mathrm{Cu}^{2+}\right]$ 的关系图 (实线由计算得到)

○示 $1.0 \times 10^{-4} \mathrm{~mol} \cdot \mathrm{dm}^{-3}, \times$ 示 $2.0 \times 10^{-4} \mathrm{~mol} \cdot \mathrm{dm}^{-3}$, ○示 $3.0 \times 10^{-4} \mathrm{~mol} \cdot \mathrm{dm}^{-3}, \Delta$ 示 $4.0 \times 10^{-4} \mathrm{~mol} \cdot \mathrm{dm}^{-3}$

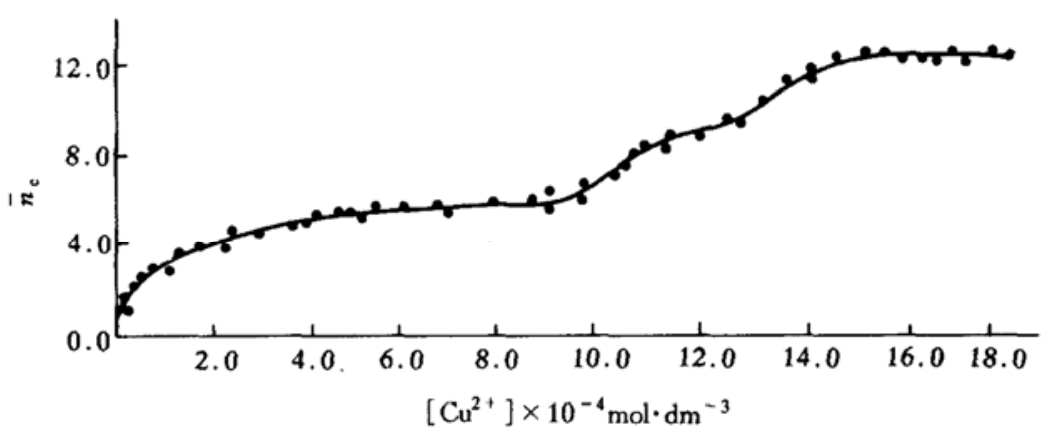

图 $2 \mathrm{BSA}$ 浓度 $2.0 \times 10^{-4} \mathrm{~mol} \cdot \mathrm{dm}^{-3}$ 时 $\mathrm{Cu}\left(\right.$ II ) $-\mathrm{BSA}$ 的 $\bar{n}_{\mathrm{c}}$ 与 $\left[\mathrm{Cu}^{2+}\right]$ 的关系图(实线由计算得到)

如用 $\bar{n}_{\mathrm{c}} /\left[\mathrm{Cu}^{2+}\right]$ 对 $\bar{n}_{\mathrm{c}}$ 作传统的 Scatchard 图(篇幅限制, 仅叙述)不难发现, 随着 $\bar{n}_{\mathrm{c}}$ 的增 加, 曲线先下降后又趋于与 $\bar{n}_{\mathrm{c}}$ 轴平行. 这说明对本文研究的体系仅用 Scatchard 作图已不能 够得到结合部位数与结合常数, 而且也说明血清白蛋白与 $\mathrm{Cu}^{2+}$ 既有专一性的结合部位, 也有 非专一性结合部位. 对于这种情况, 我们运用非线性最小二乘法拟合了逐级稳定常数值. 逐 级平衡常数遵从 Bjerrum 公式:

$$
\bar{n}=\frac{K_{1}[\mathrm{M}]+2 K_{1} K_{2}[\mathrm{M}]^{2}+\cdots+n K_{1} K_{2} \cdots K_{n}[\mathrm{M}]^{n}}{1+K_{1}[\mathrm{M}]+K_{1} K_{2}[\mathrm{M}]^{2}+\cdots+K_{1} K_{2} \cdots K_{n}[\mathrm{M}]^{n}},
$$

式中 $K_{i}(i=1,2, \cdots \cdots, n)$ 是逐级稳定常数. [M] 是金属离子的平衡浓度. 拟合偏差的值由 Hamilton R-因子估计： 


$$
R=\left[\frac{\sum_{i=1}^{n}\left(\tilde{n}_{\mathrm{p}}-\bar{n}_{\mathrm{c}}\right)^{2}}{\sum_{i=1}^{n} \bar{n}_{\mathrm{p}}{ }^{2}}\right]^{\frac{1}{2}},
$$

公式中, 脚标 $\mathrm{p}, \mathrm{c}$ 分别指实验值与计算值. 图 1 和图 2 中的实线都是对 $\bar{n}_{\mathrm{c}}$ 画的, 拟合数据得 到 20 个计量结合常数, 故取 $\vec{n}_{\mathrm{c}}=20$, 拟合结果见表 1 .

表 $1 \mathrm{Cu}($ II $)-\mathrm{HSA}$ 和 $\mathrm{Cu}$ ( II )-BSA 体系的逐级稳定常数

\begin{tabular}{|c|c|c|c|c|c|c|}
\hline \multicolumn{2}{|c|}{ 系统 } & \multicolumn{4}{|c|}{$\mathrm{Cu}($ II $)-\mathrm{HSA}$} & \multirow{2}{*}{$\frac{\mathrm{Cu} \text { ( II )-BSA }}{2.0 \times 10^{-4}}$} \\
\hline 白蛋白浓度 & & $1.0 \times 10^{-4}$ & $2.0 \times 10^{-4}$ & $3.0 \times 10^{-4}$ & $4.0 \times 10^{-4}$ & \\
\hline & $K_{1}$ & $2.53 \times 10^{4}$ & $2.42 \times 10^{4}$ & $2.42 \times 10^{4}$ & $2.42 \times 10^{4}$ & $2.35 \times 10^{4}$ \\
\hline & $K_{2}$ & $1.68 \times 10^{4}$ & $1.55 \times 10^{4}$ & $1.54 \times 10^{4}$ & $1.58 \times 10^{4}$ & $1.44 \times 10^{4}$ \\
\hline & $K_{3}$ & $1.01 \times 10^{4}$ & $9.21 \times 10^{3}$ & $9.22 \times 10^{3}$ & $1.03 \times 10^{4}$ & $8.11 \times 10^{3}$ \\
\hline & $K_{4}$ & $3.93 \times 10^{3}$ & $2.21 \times 10^{3}$ & $3.20 \times 10^{3}$ & $5.01 \times 10^{3}$ & $2.09 \times 10^{3}$ \\
\hline & $K_{5}$ & $4.55 \times 10^{3}$ & $3.94 \times 10^{3}$ & $3.73 \times 10^{3}$ & $5.50 \times 10^{3}$ & $2.75 \times 10^{3}$ \\
\hline & $K_{6}$ & $1.24 \times 10^{3}$ & $1.27 \times 10^{3}$ & $1.37 \times 10^{3}$ & $1.50 \times 10^{3}$ & $2.53 \times 10^{2}$ \\
\hline & $K_{7}$ & $1.69 \times 10^{3}$ & $2.14 \times 10^{3}$ & $6.45 \times 10^{2}$ & $1.65 \times 10^{3}$ & $1.34 \times 10^{3}$ \\
\hline & $K_{8}$ & $1.19 \times 10^{3}$ & $1.70 \times 10^{3}$ & $9.86 \times 10^{2}$ & $9.55 \times 10^{2}$ & $1.28 \times 10^{3}$ \\
\hline & $K_{9}$ & $4.1 \times 10^{2}$ & $6.26 \times 10^{2}$ & $2.75 \times 10^{2}$ & $2.96 \times 10^{2}$ & $5.36 \times 10^{2}$ \\
\hline 逐级稳定 & $K_{10}$ & $4.6 \times 10^{2}$ & $3.37 \times 10^{2}$ & $1.86 \times 10^{2}$ & $2.85 \times 10^{2}$ & $1.99 \times 10^{2}$ \\
\hline \multirow[t]{10}{*}{ 常数 } & $K_{11}$ & $1.18 \times 10^{3}$ & $9.04 \times 10^{2}$ & $7.80 \times 10^{2}$ & $9.04 \times 10^{2}$ & $6.25 \times 10^{2}$ \\
\hline & $K_{12}$ & $1.58 \times 10^{3}$ & $1.21 \times 10^{3}$ & $1.11 \times 10^{3}$ & $1.25 \times 10^{3}$ & $8.48 \times 10^{2}$ \\
\hline & $K_{13}$ & $1.56 \times 10^{3}$ & $1.22 \times 10^{3}$ & $1.15 \times 10^{3}$ & $1.29 \times 10^{3}$ & $7.98 \times 10^{2}$ \\
\hline & $K_{14}$ & $1.23 \times 10^{3}$ & $1.18 \times 10^{3}$ & $1.16 \times 10^{3}$ & $1.26 \times 10^{3}$ & $8.07 \times 10^{2}$ \\
\hline & $K_{15}$ & $5.73 \times 10^{2}$ & $9.50 \times 10^{2}$ & $1.01 \times 10^{3}$ & $1.04 \times 10^{3}$ & $8.14 \times 10^{3}$ \\
\hline & $K_{16}$ & $1.39 \times 10^{2}$ & $5.71 \times 10^{2}$ & $6.89 \times 10^{2}$ & $6.33 \times 10^{2}$ & $7.76 \times 10^{2}$ \\
\hline & $K_{17}$ & $1.06 \times 10^{2}$ & $2.90 \times 10^{2}$ & $3.92 \times 10^{2}$ & $3.12 \times 10^{2}$ & $7.10 \times 10^{2}$ \\
\hline & $K_{18}$ & $1.53 \times 10^{2}$ & $1.95 \times 10^{2}$ & $2.44 \times 10^{2}$ & $2.00 \times 10^{2}$ & $5.81 \times 10^{2}$ \\
\hline & $K_{19}$ & $1.55 \times 10^{2}$ & $1.58 \times 10^{2}$ & $1.73 \times 10^{2}$ & $1.59 \times 10^{2}$ & $3.68 \times 10^{2}$ \\
\hline & $K_{20}$ & $1.01 \times 10^{2}$ & $1.01 \times 10^{2}$ & $1.03 \times 10^{2}$ & $1.01 \times 10^{2}$ & $1.53 \times 10^{2}$ \\
\hline R-因子 & & 0.00292 & 0.00232 & 0.000333 & 0.00419 & 0.00504 \\
\hline
\end{tabular}

由表 1 可见, 处于前面的 $K$ 值 $\left(K_{1} \sim K_{5}\right)$ 远大于随后的, 这显示出 $K_{1} \sim K_{5}$ 的重要性. $\mathrm{Cu}$ ( II )-HSA 体系中第一个浓度 $\left(1.0 \times 10^{-4} \mathrm{~mol} \cdot \mathrm{dm}^{-3}\right)$ 下的 $K_{1}$ 均略大于后面 3 个较高浓度 下的 $K_{1}$ 值. 这可能与我们曾报道 ${ }^{[3,4]} \mathrm{Cu}$ ( II )-HSA 和 $\mathrm{Cu}$ ( II )-BSA 中的强结合部位均位于结 合白蛋白的 $\mathrm{N}$ 端三肽段, 且在白蛋白浓度较低时取五配位的四方雉结构而在较高浓度时取四 配位的平面四方形 ( $\mathrm{Asp}^{1}$ 上的羧基氧不再参与配位)结构有关; 而 $\mathrm{Cu}$ ( II )-BSA 体系的前 5 个 $K$ 值 $\left(K_{1} \sim K_{5}\right)$ 均较 $\mathrm{Cu}($ II $)$-HSA 体系的相应 $K$ 值略小, 可能因 BSA 中的 $\mathrm{Thr}^{2}$ 较 HSA 中 $\mathrm{Ala}^{2}$ 具有较大的位阻有关, 且可能因具较大位阻而消减了强结合部位构型变化对逐级稳定常 数的影响, 使不同浓度的 $\mathrm{Cu}$ (II)-BSA 体系的 $K_{1}$ 值基本一致. 另外, 由于 $\mathrm{Cu}^{2+}$ 与白蛋白作用 时两者浓度对白蛋白的构象是有影响的 ${ }^{[5]}$, 而 HSA 中胱氨酸的一S-S一附近的丙氨酸残基 较 BSA 的多, 这种较小的氨基酸对于 HSA 在此位置形成内聚 (高级结构) 比较有利 ${ }^{[6]}$, 表明在 同时存在 $\mathrm{Cu}^{2+}$ 的情况下, $\mathrm{HSA}$ 构象较 BSA 更易受到影响, 所以表现出不同浓度 $\mathrm{HSA}$ 与 $\mathrm{Cu}^{2+}$ 结合的 $K_{1}$ 值的差异, 而在 BSA 中, 结合的 $K_{1}$ 值却基本上与白蛋白浓度无关.

应该指出的是, Masuoka ${ }^{[7]}$ 曾报道 $\mathrm{Cu}^{2+}$ 与 $\mathrm{HSA}$ 和 BSA 结合的 $p K$ 值分别为 11.18 , 11.12 , 远远大于本文的结果. 这种结果上的显著差别, 是因为除实验条件的差别外, 更明显的 不同在于他们对白蛋白进行了脱辅基(实际上是精制)处理,这样一来某些部位被激活,参与配 
位的活性基团(如一SH) 将大大增加, 势必会造成 $K$ 值的偏大.

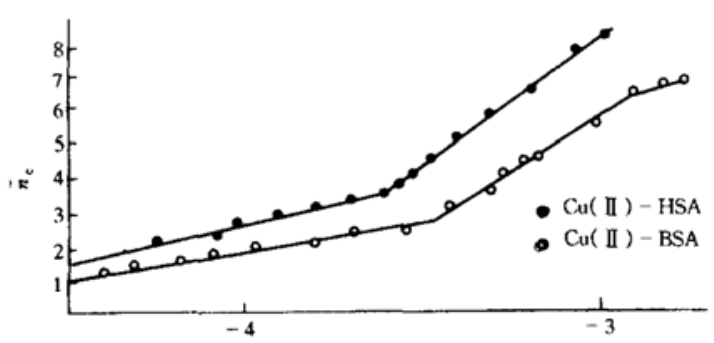

$\log \left[\mathrm{Cu}^{2+}\right]$

图 $3 \vec{n}_{\mathrm{c}}$ 对 $\log \left[\mathrm{Cu}^{2+}\right]$ 的关系图 浓度为 $2.0 \times 10^{-4} \mathrm{~mol} \cdot \mathrm{dm}^{-3}$

两体系中, $K_{1} / K_{2}$ 大致符合统计效应, 随后 的 $K_{n} / K_{n+1}$ 却偏离统计效应甚远. 这表明结合 第一个 $\mathrm{Cu}^{2+}$ 产生的配位效应不大．但它的结合 能使白蛋白发生变构效应 (Allosteric effect), 使得 随后的 $\mathrm{Cu}^{2+}$ 的结合表现出较强的配位倾向.

用 $\bar{n}_{\mathrm{c}}$ 对 $\log \left[\mathrm{Cu}^{2+}\right]$ 作图 ${ }^{[8]}$ 得图 3. 由图 3 的 两条曲线的拐点数可以看到, 两体系中结合部位 类型数不同, HSA 中的 20 个结合位置大致可分 为二类, 而 BSA 中则有三类结合部位, 这与上面 得到的两个体系的 $\bar{n}_{\text {max }}$ 值是互相支持的.

\section{$2.2 \mathrm{Cu}(\mathrm{II})$ 与 HSA 或 BSA 的结合配体分析}

$\mathrm{Cu}$ (II) 与 $\mathrm{BSA}$ 或 HSA 的结合平衡常数小于相应 $\mathrm{Zn}$ ( II ) 体系的, 而与 $\mathrm{Ni}$ (II) 体系相

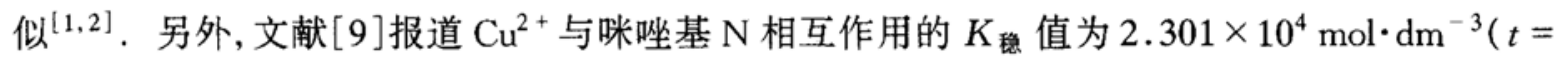
$22.5^{\circ} \mathrm{C}$ ), 这与本文中平衡常数 $K_{1}$ 类似, 更进一步支持了 $\mathrm{Cu}($ II $)$ 在 HSA 和 BSA 中的强结合 位置均位于白蛋白 $\mathrm{N}$ 端三肽段的推断. 如果自由铜离子和白蛋白的浓度继续降低 $\left(<10^{-5}\right.$ $\mathrm{mol} \cdot \mathrm{dm}^{-3}$ ), 我们推断 $K$ 略大于 $10^{4}$ 是可能的. 这种 $K$ 值变大可由白蛋白分子中的 $\mathrm{Asp}^{1}$ 羧 基氧参与配位与 $\mathrm{His}^{3}$ 咪唑基 $\mathrm{N}$ 等基团一起形成一个具有五配位构型的强结合部位得到解 释 $^{[4]}$. 只是由于实验上的困难, 我们未将浓度扩大到此范围内.

考察 $\mathrm{Cu}$ ( II )-血清白蛋白的紫外光谱图, 未在 $375 \mathrm{~nm}$ 处显示出吸收 (这是 $\mathrm{Cu}$ ( II ) 与半胱 氨酸 S 结合的证据). 而且在 $\mathrm{Zn}$ ( II )-HSA 中报道 ${ }^{[1]}$ 的 $\mathrm{Zn}$ ( II ) 诱导 HSA 交联自缔合现象在 $\mathrm{Cu}($ II ) 体系中也并未发现 (文献[1] 认为 $\mathrm{Zn}($ II ) 与 $\mathrm{S}$ 结 合, 通过半胱氨酸 $\mathrm{S}$ 形成了- $\mathrm{S}-\mathrm{Zn}-\mathrm{S}$ - 交联结构 $)$. 由此可见, 在血清白蛋白中与 $\mathrm{Cu}^{2+}$ 优先结合配位的是 $\mathrm{His}^{3}$ 的咪唑基 $\mathrm{N}$ 而不是 $\mathrm{S}$. 文献 [10]报道 HSA 和 BSA 中咪唑基分别有 16 和 17 个. 结合本实验结果, 估计大 部分 $\mathrm{Cu}^{2+}$ 是与咪唑基 $\mathrm{N}$ 配位的. 有些咪唑基可能由于 周围侧基的位阻大或位于疏水空内不利于与 $\mathrm{Cu}^{2+}$ 结 合, 而使 $\mathrm{Cu}^{2+}$ 与少部分的次要部位结合, 即形成非特异 性的较弱结合.

为了进一步了解 $\mathrm{Cu}^{2+}$ 在蛋白体系中的金属中心物 种的性质, 我们仔细分析了 Hill 系数 $n_{\mathrm{H}}$, 的定义如下:

$$
n_{\mathrm{H}}=\operatorname{dlg}\left(\frac{Y}{1-Y}\right) / \operatorname{dlg}\left[\mathrm{Cu}^{2+}\right], Y=\frac{\bar{n}}{N},
$$

其中 $N$ 为最大结合部位数, $\mathrm{Cu}$ ( II )- $\mathrm{HSA}$ 和 $\mathrm{Cu}$ ( II )BSA 的 $n_{\mathrm{H}}$ 对 $\ln \left[\mathrm{Cu}^{2+}\right]$ 点绘图分别见图 4, $n_{\mathrm{H}}$ 都在某 一自由离子浓度 $\left[\mathrm{Cu}^{2+}\right.$ ]处达到极大值 $n_{\mathrm{H}, \text { max }}$, 而 $n_{\mathrm{H}, \text { max }}$ 均近似等于 $1, n_{\mathrm{H}, \max }$ 量度在给定条件下配体结合的协 同性, 它等于配体优先结合的等同位置数 ${ }^{[11]}$. 这表明,
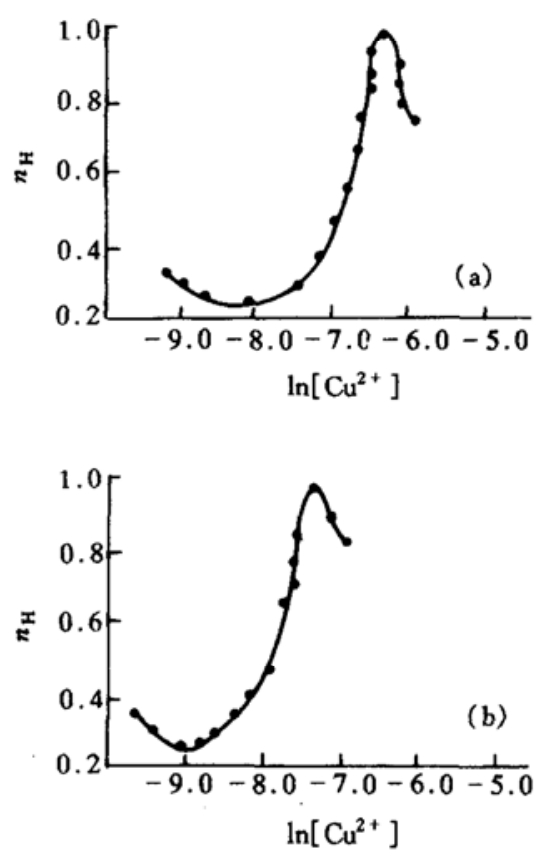

图 $4 \mathrm{Cu}\left(\right.$ II )- $\mathrm{HSA}$ 和 $\mathrm{Cu}$ ( II )-BSA 中 $n_{\mathrm{H}}$ 与 $\ln \left[\mathrm{Cu}^{2+}\right]$ 的关系图 
$\mathrm{Cu}^{2+}$ 在 HSA 和 BSA 分子中具有一个优先的结合部位, 此结合有正的协同效应 $\left(n_{\mathrm{H}}>0\right)$. 这 证实了 $\mathrm{Cu}$ ( II )-HSA和 $\mathrm{Cu}$ ( II )-BSA 体系中均具有一个强的金属结合部位的推测 ${ }^{[3.4]}$.

\section{3 铜离子与血清白蛋白结合的协同性}

根据自由能偶合可以描述配体结合的协同作用(Cooperativity)或拮抗作用(Antagonistivity), 由公式

$$
\Delta G_{x x}=\Delta G\left(p-p x_{n}\right)-n \Delta G(p-p x), \quad \Delta G=-R T \ln K,
$$

可估算结合几个配体的平均自由能偶合情况. 考虑到统计因子后, $K_{n} / K_{n+1}=$ $\frac{(N-n+1)(n+1)}{(N-n) \cdot n}=f_{n}$, 所以可用 $f_{n} K_{n+1}$ 来分析自由能偶合. $N$ 表示最大结合部位数. 利 用表 1 数据并取 $T=293 \mathrm{~K}$ 可得

$$
\Delta G_{x x}=-R T\left(\ln f_{1} K_{1} K_{2}-2 \ln K_{1}\right)=-0.806 \mathrm{~kJ} \cdot \mathrm{mol}^{-1}<0,
$$

表明第一个 $\mathrm{Cu}($ II $)$ 与 $\mathrm{HSA}$ 和 $\mathrm{BSA}$ 结合产生不太强的协同作用, 利于第二个铜离子的结合. 可能正是 $\mathrm{Cu}^{2+}$ 结合产生的这种协同作用引起的白蛋白的变构效应抑制了 $\mathrm{Cu}(\mathrm{II})$ 诱导白蛋白 的自缔合. 这一推测将在今后的工作中进一步验证.

致谢 本工作为国家自然科学基金(批准号:29271025)和广西自然科学基金资助项目.

\section{参考文献}

1 Zhou Y Q, Wang Y W, Hu X Y, et al. Equilibrium dialysis of metal-serum albumin I . Successive stability constants of $\mathrm{Zn}$ ( II )-serum albumin and the $\mathrm{Zn}^{2+}$-induced cross-linking self-association. Biophysical Chemistry, 1994, 51: 81 87

2 周永洽, 太俊哲, 梁 宏, 等. 金属-血清白蛋白的结构研究 (N)- $\mathrm{Ni}$ (II)-HSA 和 $\mathrm{Ni}$ ( II )-BSA 的新型减色效应. 高等 学校化学学报, 1996, 9:1331 1335

3 Shen P W, Zhou Y Q, Wang S Y, et al. Structural studies on metal-serum albumin I. An ultraviolet spectroscopic study of copper( II )-human serum albumin complexes. Inorg Chim Acta, 1990, 169(2): 161 166

4 周永洽、梁 宏, 申泮文, 等. 金属-血清白蛋白的结构研究 (VI)——等离子点附近 HSA 和 BSA 中 $\mathrm{Cu}$ ( II ) 和 $\mathrm{Ni}$ ( II ) 金属 中心的结构. 无机化学学报, 1992, 8(4):382 386

5 Abad C, Trueba M, Macarulla J M, et al. Structural studies of copper protein systems by Dilatomery, differential refractometry, and viscometry. An Quim Ser A, 1980, 76(2): 171 179

6 Korsenoer V M, Oratz M, Rothchild M A. Albumin Structure Functions and Uses. Oxford: Pergamon Press, 1977

7 Masuoka J, Hegenauer J, Van Dyke B R, et al. Intrinsic stoichiometric equilibrium constants for the binding of zinc( II ) to the high affinity site of serum albumin. J Biol Chem, 1993, 268(29): $21533 \sim 537$

8 Wells, J W. In: Hulme E C, ed. Receptor-Ligand Interactions, Oxford: Oxford Univ Press, 1992

9 Edsall J T, Felsenfeld G, Goodman D S, et al. The association of imidazole with the ions of zinc and cupric copper. J Am Chem Soc, 1954, 76: 3 054 3 061

10 Putnam F W. The Plasma Protein Structure, Functions and Genetic Control, 2nd ed. New York: Academic Press, 1975

11 Rubin M M, Changeux J P. On the nature of allosteric transitions: implications of non-exclusive ligand binding. J Mol Biol, 1966, 21(2): 265 274

（1996-11-12 收稿, 1997-07-25 收修改稿） 